\title{
An unusual interpodocyte cell junction and its appearance in a transplant graft kidney
}

\author{
D J HARRISON, * D JENKINS, $\dagger$ J DICK $\ddagger$
}

From the *Department of Pathology, University of Edinburgh, †Medical Renal Unit, Royal Infirmary, Edinburgh, and the $\ddagger$ Nuffield Transplantation Unit, Western General Hospital, Edinburgh

SUMMARY In a case of focal and segmental glomerulonephritis unusual cell junctions were discovered between podocytes. These most closely resembled lesions described in aminonucleoside induced nephrosis in rats and were unlike anything previously seen in our experience. Shortly after renal transplantation nephrotic syndrome recurred and biopsy specimens showed recurrent focal and segmental glomerulonephritis, with the appearance of these unusual interpodocyte junctions in the graft kidney. This may be related to circulating factors in the blood of the patient.

Focal and segmental glomerulosclerosis, with or without mesangial proliferation, ${ }^{1}$ is one of the commoner glomerular lesions to recur in transplanted graft kidneys. ${ }^{2}$ Patients present with proteinuria, sometimes within hours of transplantation, ${ }^{3}$ and may progress to renal failure, without evidence of graft rejection; circulating host factors may be responsible for disease recrudescence. ${ }^{4}$ We report a case of focal and segmental glomerulosclerosis with mesangial hypercellularity associated with a previously undescribed type of cell junction between podocytes, which recurred after transplantation and showed formation of these most unusual cell junctions.

\section{Case report}

A 62 year old woman presented in 1979 with nephrotic syndrome, her proteinuria was $15 \mathrm{~g} /$ day. There was mild renal impairment (urea $10 \mathrm{mmol} / 1$, creatinine clearance $56 \mathrm{ml} /$ minute) but no evidence of systemic disease. Serological screening, including autoantibodies, serum electrophoresis, and complement, yielded negative results. A renal biopsy was performed. This showed a focal and segmental glomerulosclerosis with a segmental increase in mesangial cellularity. No vasculitis was seen and there were only minor tubulointerstitial changes. Immunofluorescence showed IgM and C3 in sclerotic and hypercellular areas, with traces of IgG, IgA, and C4. Electron microscopy confirmed the sclerosis and mesangial increase and showed extensive loss of pedicel structure. The most striking feature was the presence of prominent junctional complexes between

Accepted for publication 8 September 1987 podocytes (fig 1), which were characterised by a thickening of the apposed plasma membranes and an

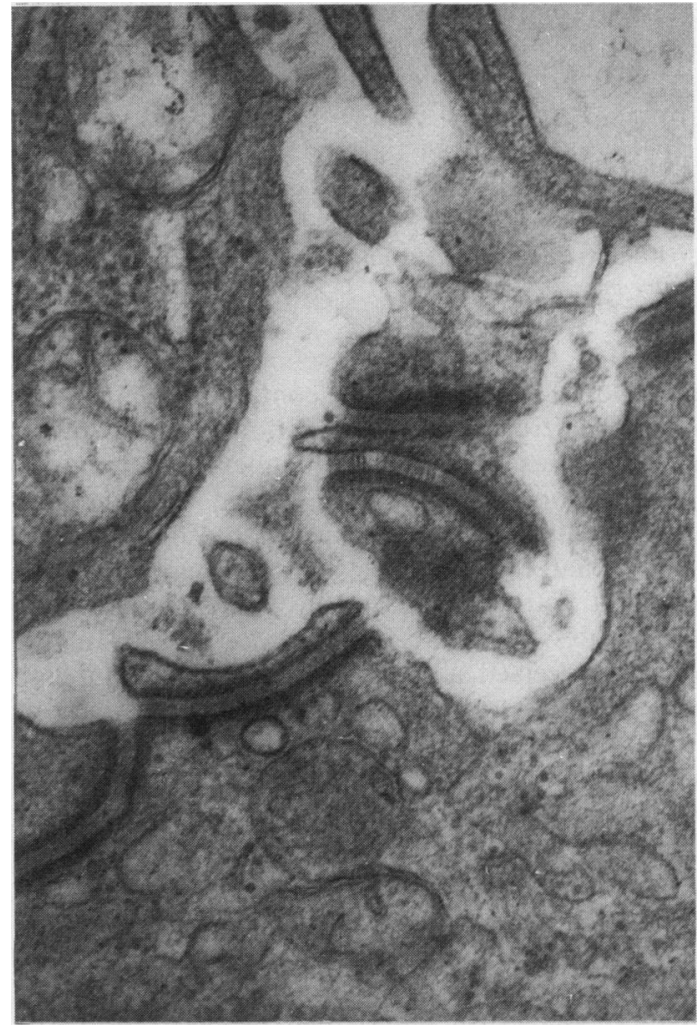

Fig 1 Electron micrograph of biopsy specimen before transplantation showing junctions between apposing podocyte cytoplasmic membranes. Note thickening of apposed membranes and fibrillary material in intercellular space. 


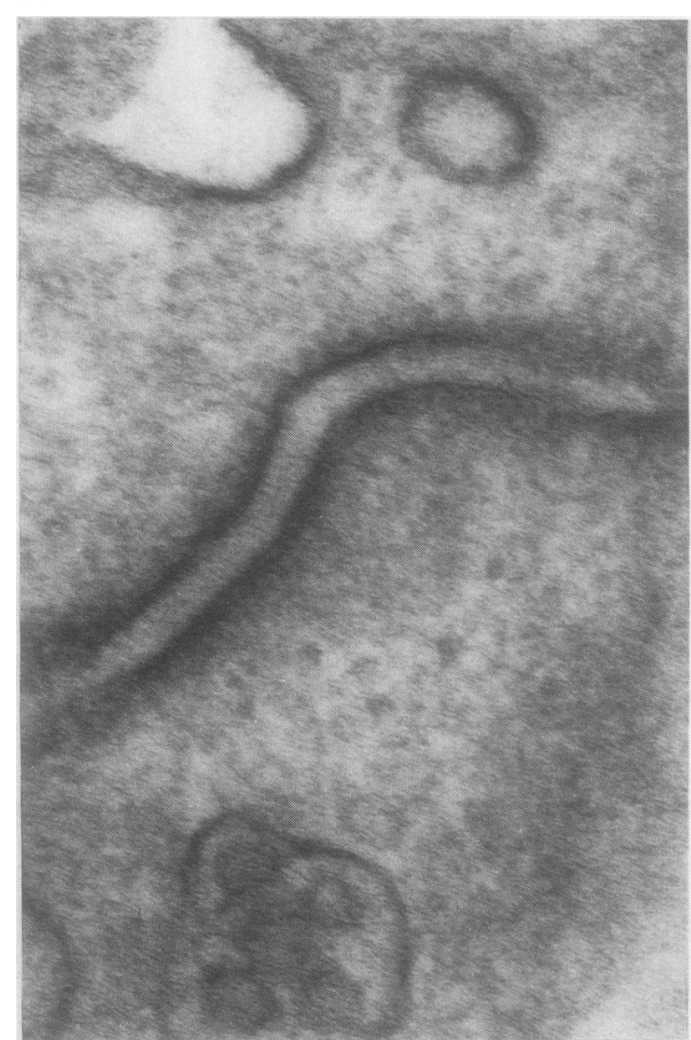

Fig 2 Electron micrograph of biopsy specimen immediately after biopsy showing thickening of apposing cytoplasmic membranes.

intercellular space of $30 \mathrm{~nm}$ containing filamentous material. There was no accumulation of tonofilaments in the cytoplasm apposed to the membrane plaques. These junctions are unlike anything we have seen before at this site and resembled desmosomes. ${ }^{5}$

Her renal function deteriorated steadily despite treatment with steroids and cycophosphamide, and haemodialysis was started in February 1980. In July 1985 she received a cadaveric renal transplant and showed good renal function immediately postoperatively. Within 16 hours her urinary output fell, the graft site was explored, and a haematoma evacuated. A renal biopsy specimen showed no histological lesion, but electron microscopy showed that in several places apposing membranes of podocytes had become thickened, although the intercellular space did not contain obvious filamentous material (fig 2). Urinary excretion improved but it soon became apparent that proteinuria had recurred and she became nephrotic. Repeat renal biopsy specimens showed recurrent focal and segmental glomerulosclerosis with mesangial proliferation, initially with no

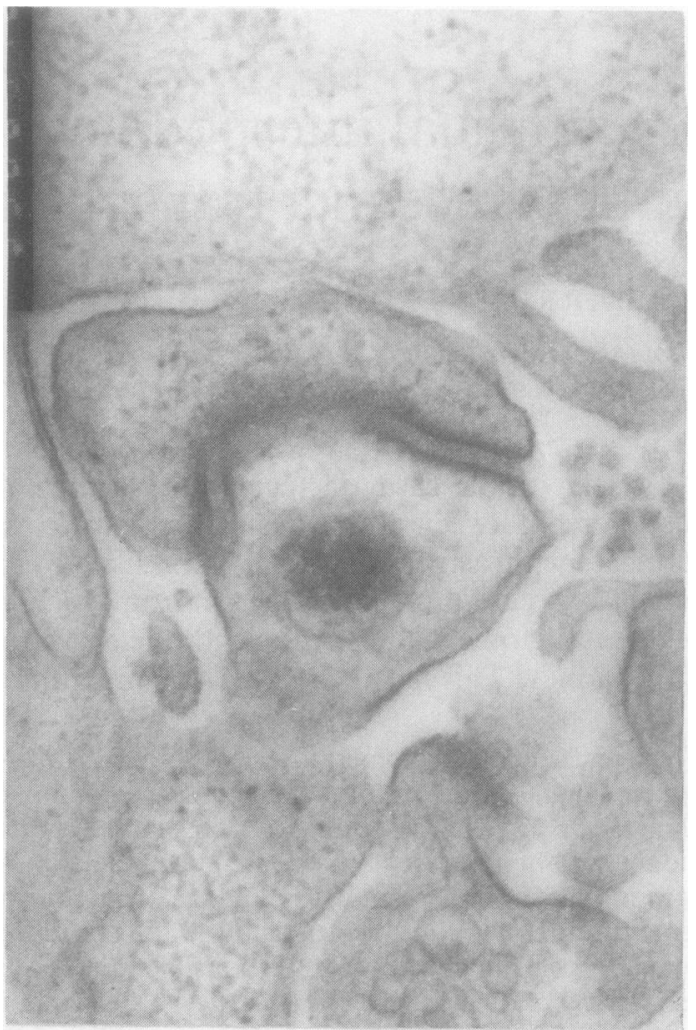

Fig 3 Graft nephrectomy electron micrograph with junctions between podocytes similar to those seen in original pretransplant biopsy specimen.

immunofluorescence positivity but later with IgM and C3 in sclerotic regions. Despite conversion from azathioprine to cyclosporin the proteinuria continued and renal function declined. Graft nephrectomy in $\overline{0}$ June 1986 showed focal and segmental glomerulosclerosis with abundant IgM and C3 in sclerotic 8 regions and traces of $\mathrm{IgG}, \mathrm{IgA}$, and $\mathrm{C4}$. Electron microscopy of all these biopsy specimens showed 9 podocyte junctions similar to, but less well defined than, the original pretransplant biopsy specimen (fig 3).

\section{Discussion}

In a review of 155 published cases of renal transplants in 140 patients with focal and segmental glomerulosclerosis the overall incidence of recurrent focal and $\underset{\mathbb{D}}{\overparen{D}}$ segmental glomerulosclerosis was $23 \%$, with a higher $\stackrel{\mathcal{C}}{+}$ incidence of recurrence in second grafts, ${ }^{2}$ although 7 there is wide variation between centres. ${ }^{6}$ The uncertain

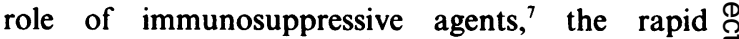
recurrence of proteinuria, ${ }^{3}$ often without positive 
immunofluorescence suggest that immune mechanisms in focal and segmental glomerulosclerosis may not be of primary importance. Indeed, focal and segmental glomerulosclerosis is associated with recurrent reflux $^{8}$ and glomerular hyperperfusion ${ }^{9}$ in the absence of any apparent immunological role.

The exact nature of these junctions is uncertain, and we are not aware of their description in human glomeruli in other cases of focal and segmental glomerulosclerosis. They most closely resemble the ultrastructural changes reported in aminonucleoside induced nephrosis in rats ${ }^{10}$ where there was elongation and displacement of the glomerular slit diaphragm away from the glomerular basement membrane. Slit diaphragms, however, have not been convincingly shown in human glomeruli. In some respects the junctions resemble desmosomes which are not normally present between podocytes. ${ }^{5}$ The appearance of desmosomes has been described in reactive synovium of rheumatoid arthritis but not in normal synovium." In desmosomes the associated intermediate filaments are normally cytokeratins ${ }^{12}$ which are not present in podocytes. ${ }^{13}{ }^{14}$

Changes in slit diaphragms in rats ${ }^{10}$ or in the sialoprotein content of glomerular basement membrane $^{15}$ result in proteinuria, leading to nephrotic syndrome. In this case a circulating factor might have precipitated ultrastructural and functional changes in the effective glomerular filtration barrier in a transplanted graft kidney, which resulted in recurrence of nephrotic syndrome.

We are grateful to Drs Lambie, MacDonald, and Anderton for permission to report this case.

\section{References}

1 Brown CB, Cameron JS, Turner DR, et al. Focal segmental glomerulosclerosis with rapid decline in renal junction (malignant FSGS). Clin Nephrol 1978;10:51-61.
2 Cameron JS. Glomerulonephritis in renal transplants. Transplantation 1982;34:237-45.

3 Hamburger J, Berger J, Hinglais N, Descamps B. New insights into the pathogenesis of glomerulonephritis afforded by the study of renal allografts. Clin Nephrol 1973;1:3-7.

4 Hoyer JR, Baij ZL, Vernier RL, Simmons RL, Majarian JS, Michael AF. Recurrence of idiopathic nephrotic syndrome after renal transplantation. Lancet 1972;ii:343.

5 Farquhar MG, Palade GE. Functional complexes in various epithelia. J Cell Biol 1963;17:373-412.

6 Currier CB, Papadopoulu Z, Helfrich GB, Diaz MH, Sulkin MD. Successful renal transplantation in focal segmental glomerulosclerosis. Transplant Proc 1980;11:49-54.

7 McPhaul JJ, London RE, Thompson AL, Mullins JD. Nephrogenic immunopathologic mechanisms and human renal transplantation-the problems of recurrent glomerulonephritis. Kidney Int 1976;10:135-8.

8 Bhatena DB, Weiss JH, Holland NH. Focal and segmental glomerulosclerosis in reflex nephropathy. Am J Med 1980;68: 886-92.

9 Bhatena DB, Julian BA, McMorrow RG, Baehler RW. Focal sclerosis of hypertrophied glomeruli in solitary functioning kidneys of humans. Am J Kidney Dis 1985;5:226-32.

10 Ryan GB, Rodewald R, Karnovsky MJ. An ultrastructural study of the glomerular slit diaphragm in amino-nucleoside nephrosis. Lab lnvest 1975;33:461-8.

11 Grimley PM, Sokoloff L. Synovial giant cells in rheumatoid arthritis. Am J Pathol 1966;49:931-54.

12 Cowin P, Mattey D, Garrod D. Distribution of desmosomal components in the tissues of vertebrates, studied by fluorescent antibody staining. J Cell Sci 1984;66:119-32.

13 Holthofer H, Miettinen A, Parasivuo R, et al. A fluorescent microscopic study with kidney-specific antibodies, anti-intermediate filament antibodies and lectins. Lab Invest 1983; 49:317-26.

14 Harrison DJ, Macdonald MK. The origin of cells in the glomerular crescent investigated by the use of monoclonal antibodies. Histopathology 1986;10:945-52.

15 Brenner BM, Hostetter TH, Humes HD. Molecular basis of proteinuria of glomerular origin. $N$ Engl $J$ Med 1982;298: 826-33.

Requests for reprints to: Dr D J Harrison, Department of Pathology, University Medical School, Teviot Place, Edinburgh EH8 9AG, Scotland. 